\title{
Sensibilidade do estímulo de tamanho I na perimetria computadorizada acromática para a detecção de defeitos glaucomatosos no campo visual: análise comparativa com as perimetrias de ondas curtas e acromática convencional (SITA)
}

\author{
Sensitivity of size I stimulus in achromatic automated perimetry for detection \\ of glaucomatous visual field defects: a comparative analysis with short \\ wavelength automated perimetry and standard automated perimetry (SITA)
}

Tessa de Cerqueira Lemos Mattos ${ }^{1}$

Niro Kasahara ${ }^{2}$

Maurício Della Paolera ${ }^{3}$

Ralph Cohen $^{4}$

Carmo Mandia Junior ${ }^{5}$

Geraldo Vicente de Almeida ${ }^{6}$

\section{RESUMO}

Objetivo: Comparar a sensibilidade e especificidade da perimetria acromática com estímulo de tamanho I (BB-I) com a perimetria acromática convencional (estratégia SITA) e a perimetria azul-amarelo (SWAP) para o diagnóstico precoce do glaucoma. Métodos: Setenta e três olhos de 73 pacientes suspeitos, glaucomatosos e normais foram submetidos às perimetrias SITA 24-2, BB-Ie SWAP. Após aplicação de critérios específicos de anormalidade para interpretação dos resultados, calculou-se a sensibilidade, a especificidade e a área sob a curva ROC (Receiver Operating Characteristic) para cada um dos exames. As áreas sob as curvas ROC foram comparadas aos pares com o teste Z univariável e significância 5\%. Resultados: O BB-I demonstrou maior sensibilidade (100\%) e menor especificidade $(75,7 \%)$. O SWAP revelou menor sensibilidade $(69,4 \%)$, porém maior especificidade $(89,2 \%)$. A comparação pareada entre as curvas ROC não revelou diferença estatisticamente significante entre as três técnicas perimétricas estudadas. Conclusões: O BB-I revelou-se eficaz na detecção de defeitos precoces de campo visual no glaucoma. Esta técnica soma-se ao armamentário semiológico disponível para o diagnóstico funcional precoce da neuropatia óptica glaucomatosa.

Descritores: Perimetria; Glaucoma; Sensibilidade e especificidade; Campo visual

Trabalho realizado no setor de Glaucoma da Faculdade de Ciências Médicas da Santa Casa de São Paulo FCMSCSP - São Paulo (SP) - Brasil.

Fellow do setor de Retina e Vítreo do Departamento de Oftalmologia da Universidade Federal de São Paulo UNIFESP - São Paulo (SP) - Brasil.

Médico Assistente do setor de Glaucoma da Faculdade de Ciências Médicas da Santa Casa de São Paulo FCMSCSP - São Paulo (SP) - Brasil.

Médico Assistente e Chefe do setor de Glaucoma da FCMSCSP - São Paulo (SP) - Brasil.

Professor Adjunto do Departamento de Oftalmologia da FCMSCSP - São Paulo (SP) - Brasil.

Professor Assistente do Departamento de Oftalmologia da FCMSCSP - São Paulo (SP) - Brasil.

${ }^{6}$ Professor Adjunto do Departamento de Oftalmologia da FCMSCSP - São Paulo (SP) - Brasil.

Endereço para correspondência: Niro Kasahara. Rua São Mauro, 292 - São Paulo (SP) CEP 02526-050

Recebido para publicação em 31.07.2006

Última versão recebida em 13.05.2007

Aprovação em 13.12.2007

\section{INTRODUÇÃO}

O glaucoma é uma das principais causas de cegueira irreversível no mundo. Estima-se que 67 milhões de pessoas sejam acometidas por esta doença $^{(1)}$. A semiologia do glaucoma é baseada na medida da pressão intraocular, na avaliação do disco óptico e em testes psicofísicos de avaliação funcional (perimetria computadorizada).

Dentre os testes de avaliação funcional no glaucoma, a perimetria computadorizada acromática, ou seja, estímulo branco sobre fundo branco, é considerada o exame padrão para o diagnóstico, acompanhamento e estadiamento da gravidade da doença. Entretanto, essa técnica não permite o diagnóstico precoce da lesão glaucomatosa, pois as primeiras alterações 
perimétricas somente são detectadas quando cerca de 30 a $40 \%$ das fibras nervosas da retina foram lesadas ${ }^{(2-3)}$.

Com o intuito de diagnosticar o glaucoma mais precocemente, novos testes de avaliação funcional têm sido desenvolvidos, como a perimetria de freqüência dupla (FDT) e a perimetria computadorizada de ondas curtas (SWAP).

O FDT avalia especificamente as células My, um subgrupo das células magnocelulares que correspondem a menos de $10 \%$ das células ganglionares da retina e apresentam a propriedade de resposta visual não linear ${ }^{(4)}$. O FDT revelou-se altamente sensível e específico no diagnóstico do glaucoma, sendo capaz de detectar defeitos perimétricos antes da perimetria convencional ${ }^{(5)}$. Também mostrou ser mais sensível que a polarimetria de varredura a laser e fotografias da camada de fibras nervosas da retina ${ }^{(6)}$. Não obstante, faltam estudos longitudinais para corroborar essas observações ${ }^{(7)}$.

Outro método de avaliação funcional desenvolvido com o objetivo de detectar defeitos glaucomatosos precocemente é o SWAP ou perimetria azul - amarelo. A SWAP permite isolar e medir a função das células biestratificadas B-Y mediante estímulo azul projetado sobre o fundo amarelo. Representam $9 \%$ das células ganglionares da retina e acredita-se que sejam seletivamente lesadas na fase inicial do glaucoma ${ }^{(8)}$. A SWAP é capaz de detectar defeitos perimétricos até cinco anos antes que a perimetria acromática convecional, além de detectar a progressão do defeito mais precocemente ${ }^{(8)}$. Outra hipótese sugere que, como a SWAP avalia uma fração específica das células ganglionares, evita-se o fenômeno da redundância. Ela possui as desvantagens de ser um exame demorado, mais sensível às alterações de densidade do cristalino e apresentar maior flutuação entre os testes ${ }^{(8)}$.

As novas técnicas apresentadas acima possuem custos elevados, limitando assim a sua utilização em nosso meio. Além disso, são técnicas em evolução, que rapidamente podem tornar-se ultrapassadas com a chegada de novos programas. Desta forma, é preocupante investir num equipamento não bem estabelecido na prática clínica, que possa vir a tornar-se obsoleto em poucos anos.

A perimetria convencional utiliza um estímulo de tamanho III de Goldmann na pesquisa do campo visual. Poucos estudos relataram que o estímulo de tamanho I demonstrou maior sensibilidade que o estímulo de tamanho III na detecção de escotomas rasos podendo, por isso, ser mais útil no diagnóstico precoce do glaucoma ${ }^{(9-10)}$. Sua principal vantagem é a utilização do mesmo instrumento da perimetria convecional, dispensando a aquisição de outro aparelho.

O objetivo deste estudo foi avaliar a sensibilidade e a especificidade da perimetria acromática com estímulo de tamanho I (BB-I) no diagnóstico precoce do glaucoma e compará-la com a perimetria acromática convencional (SITA 24-4) e a perimetria azul-amarela (SWAP).

\section{MÉTODOS}

\section{Amostra e critérios de seleção}

O desenho do estudo foi transversal e incluiu 73 pacientes, divididos em três grupos: um grupo de indivíduos normais (25 indivíduos), um grupo de pacientes com glaucoma leve (24 pacientes) e um grupo de indivíduos com suspeita de glaucoma (24 pacientes).

Os pacientes foram selecionados do Departamento de Oftalmologia da Santa Casa de São Paulo de acordo com os seguintes critérios de inclusão: idade entre 40 e 60 anos, acuidade visual corrigida de 0,8 ou maior, vícios de refração menores que $\pm 4,00 \mathrm{DE}$ e/ou $\pm 2,50 \mathrm{DC}$, ausência de catarata ou qualquer alteração de transparência dos meios ópticos e ausência de cicatrizes na retina que mimetizassem defeitos perimétricos glaucomatosos. Suspeita de glaucoma foi definida como escavação do disco óptico maior que 0,7 ou assimetria maior que 0,2 entre os olhos e perimetria SITA 24-2 normal. Glaucoma leve foi definido em pacientes que apresentassem defeitos localizados ("notch") ou difusos (aumento concêntrico da escavação) na rima neural e defeitos correspondentes na perimetria acromática convencional. Foram incluídos apenas pacientes portadores de glaucoma leve que ao exame perimétrico apresentassem: MD (mean deviation) menor que - $6 \mathrm{~dB}$, menos de $25 \%$ dos pontos com p<5\% e menos de $15 \%$ dos pontos com $\mathrm{p}<1 \%$ no gráfico "pattern deviation", nenhum ponto dentro dos $5^{\circ}$ centrais com sensibilidade menor que $15 \mathrm{~dB}$. Os indivíduos foram considerados normais se ao exame oftalmoscópico apresentassem relação escavação/disco até 0,4 em ambos os olhos e pressão intra-ocular $<21 \mathrm{mmHg}$.

\section{Procedimentos}

O estudo foi aprovado pelo Comitê de Ética da Santa Casa de São Paulo e os pacientes formalizaram o consentimento para participar no estudo que seguiu as diretrizes de ética em pesquisa da Resolução 196/96 do Conselho Nacional de Saúde - Ministério da Saúde. Os pacientes que preencheram os critérios de inclusão foram submetidos à avaliação oftalmológica com medida de acuidade visual corrigida (tabela de Snellen), biomicroscopia do segmento anterior, tonometria com tonômetro de aplanação de Goldmann, biomicroscopia do disco óptico com lente de Volk 78D e três diferentes testes perimétricos. O instrumento utilizado para a pesquisa do campo visual foi o Humphrey Field Analyzer II, modelo 750 (Zeiss - Humphrey Systems, Dublin, CA, EUA). O primeiro exame realizado foi a perimetria acromática convencional (programa SITA 24-2), seguido pela perimetria azul-amarelo (central 24-2 full-threshold) e pela perimetria acromática com estímulo tamanho I de Goldmann (central 24-2 full-threshold). Antes da realização da perimetria azul-amarelo, todos os pacientes passaram por um período de adaptação olhando para cúpula amarela por cinco minutos. Os exames perimétricos foram realizados por um único examinador experiente, após correção óptica adequada.

Escolheu-se um olho de cada paciente de forma aleatória nos indivíduos normais e nos pacientes suspeitos e glaucomatosos o olho estudado foi escolhido conforme os critérios de inclusão. Os três testes perimétricos foram realizados num período entre 1 e 7 dias. Os resultados de exames não confiáveis, isto é, com mais de $20 \%$ de perda de fixação e/ou mais que 
144 Sensibilidade do estímulo de tamanho I na perimetria computadorizada acromática para a detecção de defeitos glaucomatosos no campo visual: análise comparativa com as perimetrias de ondas curtas e acromática convencional (SITA)

$33 \%$ de falso positivos e/ou falso negativos, foram repetidos por uma segunda vez. Persistindo-se a não confiabilidade, o paciente era excluído do estudo. Todos os resultados de exames positivos foram confirmados num segundo teste perimétrico, sendo este último utilizado para análise no estudo.

\section{Interpretação dos resultados}

Foram utilizados critérios específicos para definir-se o defeito glaucomatoso mínimo na interpretação dos resultados dos exames perimétricos. Na perimetria convencional (SITA 24-2), foram utilizados os critérios de Anderson modificados, a saber, presença de 3 ou mais pontos no gráfico de "pattern deviation" deprimidos com $\mathrm{p}<5 \%$, sendo um deles com $\mathrm{p}<1 \%$; GHT fora dos limites normais e PSD diminuído à significância de $5 \%{ }^{(11)}$. No exame da perimetria acromática com o estímulo I, o defeito glaucomatoso foi definido pela presença de 3 ou mais pontos com sensibilidade diminuída em pelo menos $7 \mathrm{~dB}$, conforme observados no gráfico "depth defect", ou a presença de dois ou mais pontos com sensibilidade diminuída em pelo menos $8 \mathrm{~dB}$. Estes critérios foram definidos com base no estudo de Zalta \& Burchfield ${ }^{(9)}$. Para a perimetria azul-amarelo, foram utilizados os critérios de Polo para defeito perimétrico, a saber, grupo de 4 ou mais pontos no "total deviation" com sensibilidade diminuída a significância de $5 \%$ ou grupo de 3 ou mais pontos no "total deviation" com sensibilidade diminuída a significância de $1 \%{ }^{(12)}$. Foram utilizados 52 dos 54 pontos pesquisados em cada exame (excluídos os 2 pontos correspondentes à mancha cega).

\section{Análise estatística}

Para comparar a capacidade de cada exame para diferenciar indivíduos normais de glaucomatosos, foram calculadas a sensibilidade, a especificidade e a curva ROC (Receiver Operating Characteristic) para cada teste. A curva ROC é um gráfico traçado entre a sensibilidade no eixo Y e, no eixo Y, 1 - a especificidade. Quanto maior a precisão do teste diagnóstico, mais próximo será o desenho da curva do canto superior esquerdo do gráfico, ao passo que, num teste sem valor diagnóstico, o desenho da curva coincidirá com uma linha diagonal traçada entre o canto inferior esquerdo e superior direito do gráfico. As áreas sob as curvas de cada exame foram calculadas e comparadas aos pares, isto é, SWAP e BB-I, SWAP e SITA, e BB-I e SITA com o teste Z univariável e significância a 5\%. Um teste perfeito apresenta a área sob a curva igual a 1, enquanto, um teste sem valor diagnóstico apresenta uma área de 0,5. Para cada sub-grupo de pacientes, construiu-se um diagrama de Euler (ou diagrama de Venn) para ilustrar o número de indivíduos diagnosticados por cada um dos três métodos perimétricos. $\mathrm{Na}$ análise dos pacientes suspeitos, estabeleceu-se o padrão-ouro a SWAP, isto é, a presença de defeito neste exame confirmaria o diagnóstico funcional de glaucoma. Nos pacientes normais e glaucomatosos, para análise dos resultados apresentados nos diagramas de Venn, estabeleceu-se a SITA como padrão-ouro.

\section{RESULTADOS}

As características demográficas dos 73 indivíduos incluídos no estudo são apresentadas na tabela 1 . Observou-se que a maioria dos pacientes era do sexo feminino e a proporção entre as etnias foi semelhante.

O tempo de realização da perimetria SITA foi de 5,7 $\pm 1,0$ minutos, notavelmente menor que o SWAP e o BB-I que demoraram 12,1 $\pm 1,2$ e 12,2 $\pm 1,5$ minutos, respectivamente. A (Figura 1A) mostra o exemplo do impresso do exame perimétrico com o estímulo de tamanho I de um paciente com defeito tipicamente glaucomatoso e seus correspondentes na SWAP (Figura 1B) e SITA (Figura 1C).

As áreas sob a curva ROC, a sensibilidade e especificidade de cada teste estão descritas na tabela 2. A BB-I foi a mais sensível dos três exames e a SWAP foi a mais específica.

A tabela 3 mostra a comparação aos pares entre as curvas ROC dos três testes. Não foi constatada diferença estatisticamente significante entre elas.

A figura 2 mostra o número de indivíduos suspeitos diagnosticados como glaucomatosos em cada exame. Constatouse que dos 24 indivíduos suspeitos, 18 foram rotulados como glaucomatosos em pelo menos um dos três exames. Em apenas quatro indivíduos os três testes concordaram com o diagnóstico. Nenhum paciente da amostra teve o diagnóstico de glaucoma confirmado exclusivamente pela SWAP.

A figura 3 mostra o número de indivíduos glaucomatosos diagnosticados como tal em cada teste perimétrico. Surpreendentemente, dos 24 pacientes glaucomatosos, quatro não foram detectados pelo SWAP.

A figura 4 mostra o número de indivíduos normais diagnosticados como glaucomatosos (falso-positivos) em cada exame. O BB-I foi o teste com maior número de falso-positivos.

\section{DISCUSSÃO}

Nosso estudo revelou sensibilidade do BB-I (100\%) superior à estratégia SITA $(86,1 \%)$ e à $\operatorname{SWAP}(69,4 \%)$ no diagnóstico precoce do glaucoma, confirmando resultados de outros estudos.

\begin{tabular}{|c|c|c|c|c|}
\hline Variável & $\begin{array}{c}\text { Normais } \\
(n=25)\end{array}$ & $\begin{array}{l}\text { Suspeitos } \\
(n=24)\end{array}$ & $\begin{array}{l}\text { Glaucomatosos } \\
(n=24)\end{array}$ & $\begin{array}{c}\text { Total } \\
(n=73)\end{array}$ \\
\hline Idade* (anos) & $47,3 \pm 6,0$ & $47,4 \pm 8,3$ & $54,5 \pm 4,8$ & $49,7 \pm 7,3$ \\
\hline $\mathrm{AV}^{*}$ & $0,99 \pm 0,0$ & $0,93 \pm 0,0$ & $0,95 \pm 0,1$ & $0,96 \pm 0,0$ \\
\hline Sexo (M:F) & $2: 23$ & 12:12 & 9:15 & $23: 50$ \\
\hline Etnia Branca & 6 & 8 & 12 & 26 \\
\hline Negra & 12 & 6 & 5 & 23 \\
\hline Outras & 7 & 10 & 7 & 24 \\
\hline
\end{tabular}


Sensibilidade do estímulo de tamanho I na perimetria computadorizada acromática para a detecção de defeitos glaucomatosos no campo visual: 145 análise comparativa com as perimetrias de ondas curtas e acromática convencional (SITA)

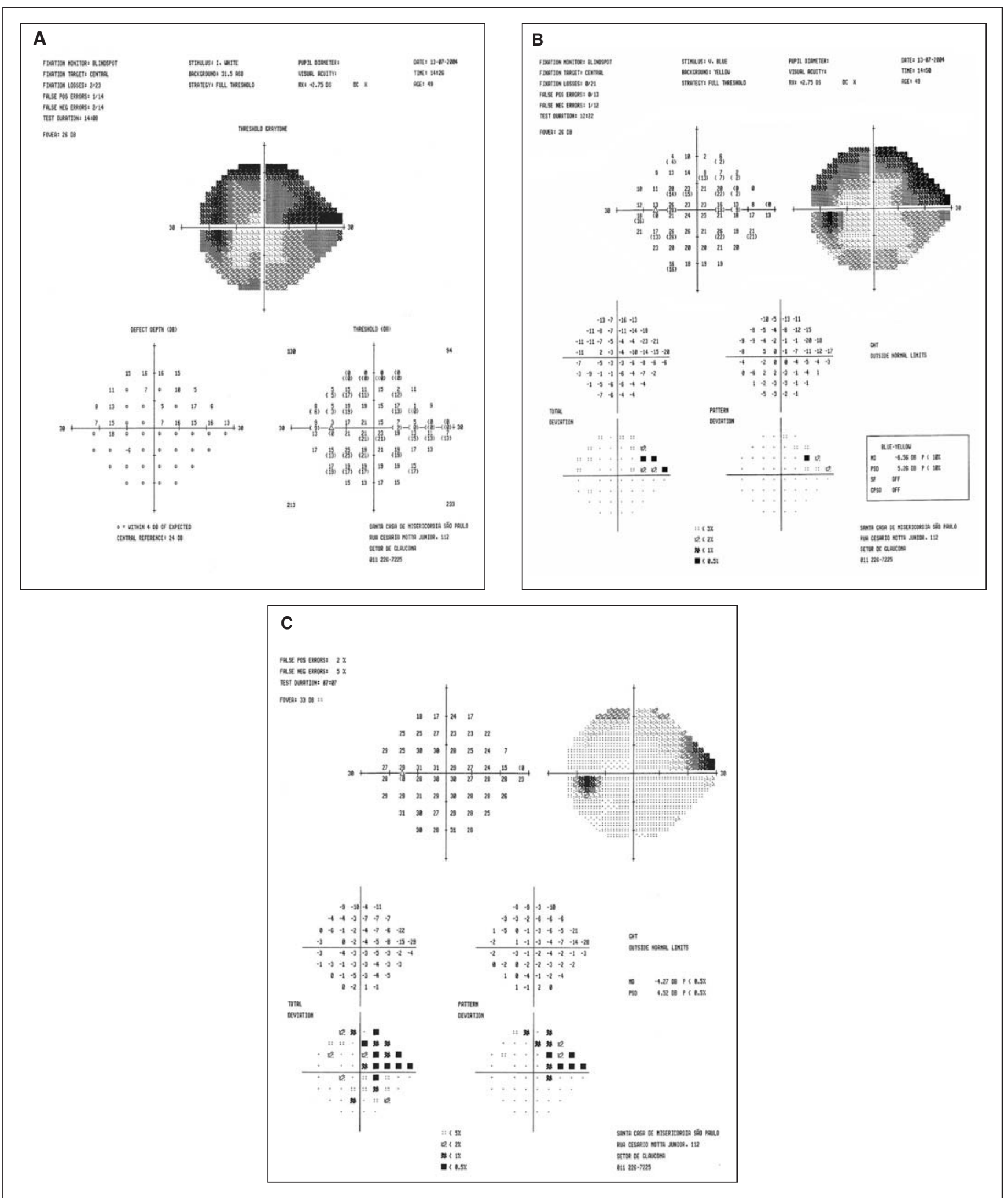

Figura 1 - Exemplo de um paciente com glaucoma primário de ângulo aberto em estádio inicial mostrando a correspondência do defeito de campo visual nos três exames perimétricos: A) Perimetria acromática com estímulo de tamanho I; B) Perimetria azul-amarelo; C) Perimetria acromática convencional com a estratégia SITA. 
146 Sensibilidade do estímulo de tamanho I na perimetria computadorizada acromática para a detecção de defeitos glaucomatosos no campo visual: análise comparativa com as perimetrias de ondas curtas e acromática convencional (SITA)

Zalta \& Burchfield investigaram a influência do tamanho do estímulo na detecção de defeitos perimétricos em 82 olhos com diagnóstico de glaucoma leve, suspeita de glaucoma e olhos normais. Os autores basearam-se no gráfico "depth defect", um gráfico de valores de sensibilidade "normal" derivado matematicamente. Neste modelo matemático, o perímetro calcula o limiar de sensibilidade para cada ponto do campo visual reduzindo $0,4 \mathrm{~dB}$ (no BB-I) ou $0,31 \mathrm{~dB}$ (na estratégia com estímulo de tamanho III) para cada grau de excentricidade a

Tabela 2. Áreas sob a curva ROC, sensibilidade e especificidade de cada exame

\begin{tabular}{|c|c|c|c|}
\hline Teste & Área (IC 95\%) & Sensibilidade & Especificidade \\
\hline SITA & $0,822(0,751-0,902)$ & 86,1 & 78,4 \\
\hline BB-I & $0,878 \quad(0,781-0,943)$ & 100,0 & 75,7 \\
\hline SWAP & $0,793(0,682-0,879)$ & 69,4 & 89,2 \\
\hline \multicolumn{4}{|c|}{$\begin{array}{l}\text { IC } 95 \%=\text { intervalo de confiança de } 95 \% \text {; SITA= perimetria acromática conven- } \\
\text { cional; BB-I= perimetria acromática com a mira I de Goldmann; SWAP= } \\
\text { perimetria azul-amarelo } \\
\text { Fonte: Departamento de Oftalmologia - Santa Casa de São Paulo, } 2004\end{array}$} \\
\hline
\end{tabular}

Tabela 3. Comparação pareada entre as curvas ROC dos três exames

$\begin{array}{lccc}\text { Par de testes } & \text { Diferença (IC 95\%) } & \text { Erro padrão } & \boldsymbol{P} \\ \text { SWAP e BB-I } & 0,085(-0,042 \text { a } 0,212) & 0,065 & 0,188 \\ \text { SWAP e SITA } & 0,029(-0,091 \text { a } 0,149) & 0,061 & 0,632 \\ \text { BB-I e SITA } & 0,056(-0,071 \text { a } 0,183) & 0,065 & 0,387\end{array}$

IC 95\%= intervalo de confiança de 95\%; SITA= perimetria acromática convencional; BB-I= perimetria acromática com o estímulo I de Goldmann; SWAP= perimetria azul-amarelo

Fonte: Departamento de Oftalmologia - Santa Casa de São Paulo, 2004

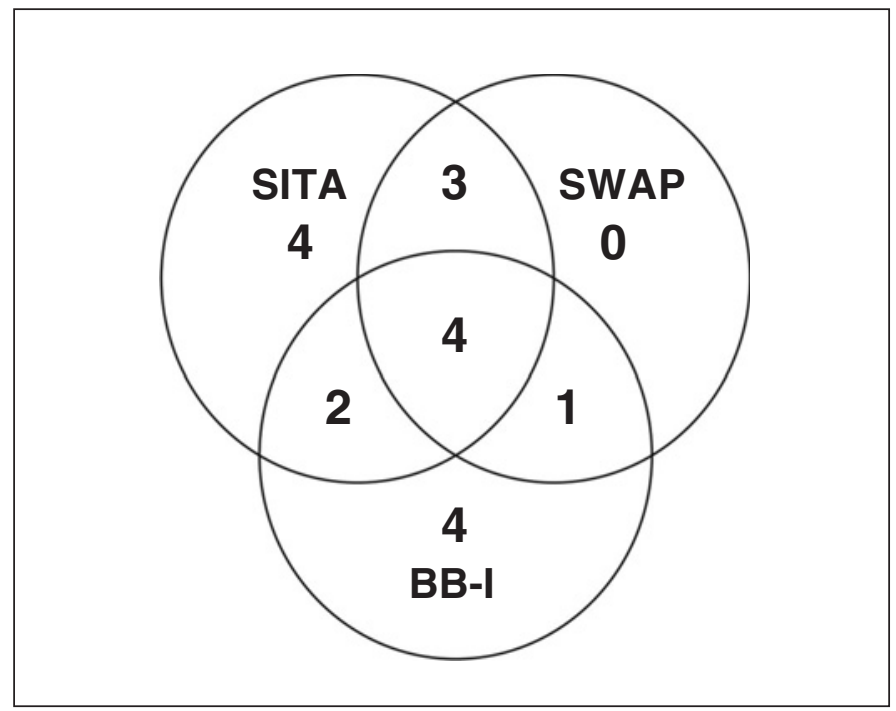

Figura 2 - Diagrama de Venn mostrando a distribuição dos 18 pacientes do grupo de suspeitos diagnosticados como glaucomatosos em pelo menos um dos exames perimétricos (SITA= perimetria convencional; BB-I= perimetria acromática com estímulo tamanho I; SWAP= perimetria azul-amarelo) partir do limiar foveal conhecido de cada faixa etária. Baseados neste banco de dados normal matematicamente calculado, os autores constataram que o estímulo de tamanho I revelou sensibilidade significantemente maior na detecção de escotomas pequenos e rasos localizados no campo visual central. Os resultados obtidos com o estímulo de tamanho I foram reprodutíveis e independentes da idade do paciente ${ }^{(9)}$.

Yamada et al. investigaram a efetividade do uso do estímulo tamanho I na detecção de defeitos do campo visual e a somação espacial (III-I) para as alterações perimétricas glaucomatosas $^{(10)}$. Baseados na sensibilidade média (MS) e desvio

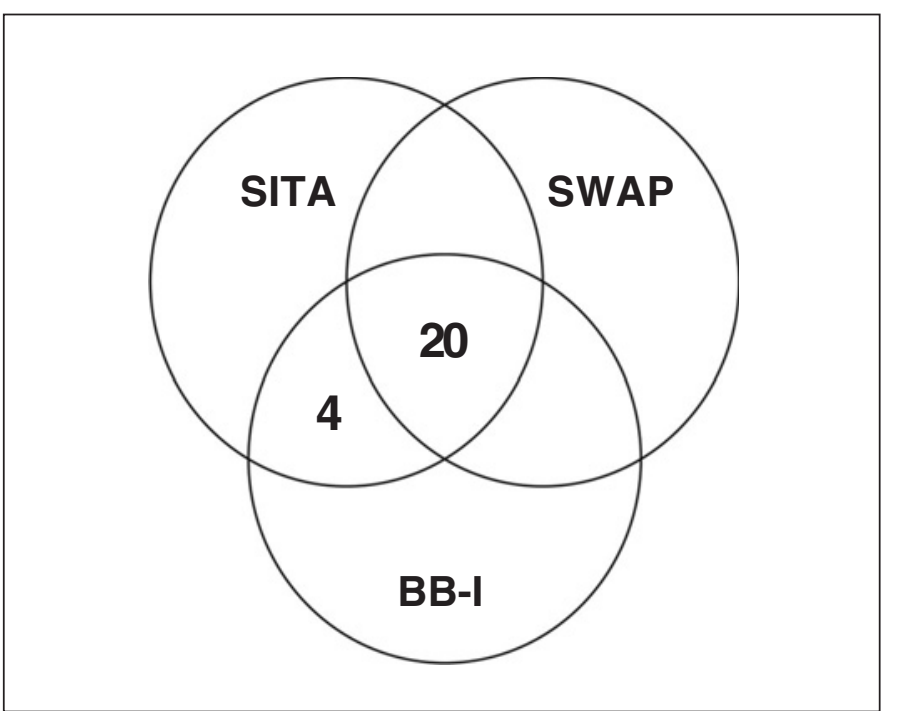

Figura 3 - Diagrama de Venn mostrando a distribuição dos 24 indivíduos glaucomatosos diagnosticados como tal em cada exame perimétrico (SITA= perimetria convencional; $\mathrm{BB}-\mathrm{I}=$ perimetria acromática com estímulo tamanho I; SWAP= perimetria azul-amarelo)

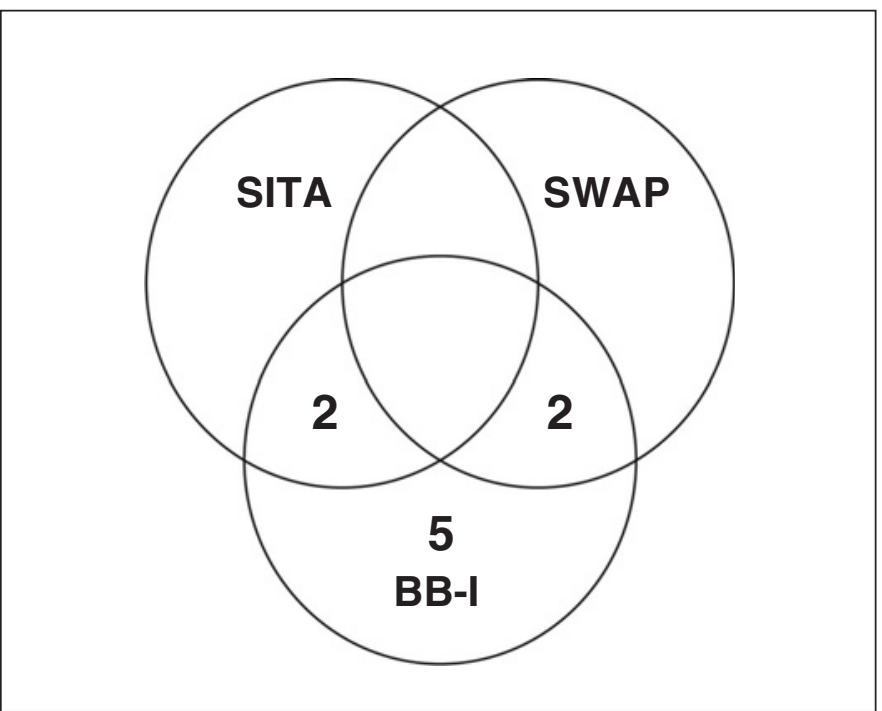

Figura 4 - Diagrama de Venn mostrando a distribuição dos 9 indivíduos normais diagnosticados como glaucomatosos em cada exame perimétrico $(\mathrm{SITA}=$ perimetria convencional; $\mathrm{BB}-\mathrm{I}=$ perimetria acromática com estímulo tamanho I; SWAP= perimetria azul-amarelo) 
padrão (SD) de cada ponto do campo visual dos indivíduos normais, o escore- $\mathrm{Z}$ da sensibilidade retínica $(\mathrm{Zi}=[\mathrm{Xi}-\mathrm{MS}] /$ $\mathrm{SD}$; onde $\mathrm{Xi}=$ valor medido) e a somação espacial (diferença na sensibilidade retínica entre o estímulo tamanho III e tamanho I) foram calculados para os exames de campo visual dos pacientes glaucomatosos. O escore-z médio na sensibilidade retínica para a mira III foi menor que o estímulo I nas áreas anormais. Nas áreas normais do campo visual, o escore-z para a mira I foi significantemente menor do que para a mira III. A somação espacial aumentava proporcionalmente ao aumento da sensibilidade pesquisada com o estímulo III. Os autores concluíram que o estímulo de tamanho I é mais sensível que o estímulo tamanho III na detecção de anormalidades em áreas do campo visual com defeitos leves e sugeriram a perimetria com mira I como método diagnóstico suplementar na detecção e acompanhamento de defeitos precoces do campo visual no glaucoma $^{(10)}$.

Nosso estudo diferiu dos trabalhos pregressos nos métodos empregados. Não calculamos a somação espacial nem o escore-z na nossa análise, porém, definimos critérios específicos de anormalidades para a perimetria BB-I, não arbitrários, mas baseados nos resultados de Zalta \& Burchfield. A aplicação de critérios específicos de anormalidades para interpretação dos resultados da perimetria é rotineiro na prática clínica, como os critérios de Anderson para a perimetria convencional e os critérios definidos por Polo na SWAP ${ }^{(11-12)}$. A definição de critérios específicos para a BB-I visou facilitar a interpretação dos resultados do teste para a realização do estudo, mimetizando a prática clínica diária. Além disso, sua transposição para a aplicação clínica torna-se mais fácil e imediata.

Por que a BB-I mostrou-se mais sensível no diagnóstico precoce do glaucoma em comparação à SITA e SWAP? Gramer et al. estudaram a influência do tamanho do estímulo da perimetria computadorizada nos defeitos de campo visual de pacientes glaucomatosos ${ }^{(13)}$. Em 12 olhos, os autores compararam os estímulos de tamanho I e III e, em 18 olhos os estímulos III e V no perímetro Octopus. Os valores médios de sensibilidade nas áreas acometidas do campo visual foram de 6 a $10 \mathrm{~dB}$ mais altas com o estímulo III do que com o I e igualmente maiores com o estímulo V em comparação com o III. Os autores concluíram que com a utilização de estímulos maiores, pequenos escotomas podem passar despercebidos e os defeitos identificados parecem menores e menos densos. A SWAP utiliza o estímulo tamanho V $\left(64 \mathrm{~mm}^{2}\right)$ de Goldmann, consideravelmente maior que estímulo I $\left(0,25 \mathrm{~mm}^{2}\right)$ do teste BB-I, explicando a maior sensibilidade deste último, independentemente da subpopulação de células ganglionares pesquisadas. A maior sensibilidade do SWAP na detecção de defeito perimétrico precoce no glaucoma tem sido contestada. Soliman et al. avaliaram a sensibilidade e especificidade da SWAP, FDT e da perimetria acromática convencional (SAP) em indivíduos glaucomatosos, suspeitos e hipertensos oculares ${ }^{(14)}$. No grupo de glaucomatosos e suspeitos, o número total de pontos anormais na SWAP foi significantemente menor que o de pontos da SAP e FDT.
Os autores concluíram que a SWAP na sua condição atual é notavelmente menos eficiente que o FDT e a SAP na detecção de defeitos perimétricos.

Apesar da maior sensibilidade, a BB-I apresentou, em contrapartida, menor especificidade e maior número de falsos positivos (Figura 4). Isto traz implicações práticas. Não se pode confiar exclusivamente num único exame para o diagnóstico funcional de glaucoma nos casos suspeitos. A repetição do teste é imperativa e sua interpretação com outros dados semiológicos é fundamental para nortear o raciocínio clínico.

A aplicação da BB-I é vantajosa principalmente nos países subdesenvolvidos, onde a aquisição dos novos equipamentos utilizados no diagnóstico precoce do glaucoma é impedida em função das dificuldades econômicas e limitações de caráter orçamentárias. A BB-I está disponível inclusive no perímetro Humphrey série 600, a versão mais antiga do equipamento. Em contra-partida, a principal desvantagem da perimetria BB-I é a inexistência de um banco de dados normal de fato. $\mathrm{O}$ gráfico "depth defect" utilizado na análise dos resultados neste estudo é um gráfico de valores de sensibilidade "normal" derivado matematicamente. Neste modelo matemático, o perímetro calcula o limiar de sensibilidade para cada ponto do campo visual reduzindo $0,4 \mathrm{~dB}$ para cada grau de excentricidade a partir do limiar foveal conhecido de cada faixa etária, sendo portanto, apenas um substituto teórico para dados normativos reais ${ }^{(9)}$.

Este estudo apresenta algumas limitações óbvias. Primeiramente, o desenho transversal não permite a confirmação diagnóstica absoluta quando se comparam diferentes testes sem um padrão ouro. Neste trabalho, estabeleceu-se a SWAP como o padrão ouro nos casos suspeitos e a perimetria convencional com a avaliação do disco óptico nos pacientes normais e glaucomatosos. Um estudo prospectivo seria o ideal para confirmação da habilidade da BB-I no diagnóstico do glaucoma. Segundo, nossa amostra apresentou o dobro de indivíduos do sexo feminino $(68,5 \%)$ em relação ao masculino $(31,5 \%)$. Isto pode ter introduzido um viés de seleção de amostra. Entretanto, não acreditamos que esse fato comprometa a validade externa do estudo, uma vez que não é conhecida qualquer diferença entre os gêneros masculino e feminino na patogenia do glaucoma primário de ângulo aberto.

\section{CONCLUSÃO}

A perimetria acromática com o estímulo tamanho I de Goldmann revelou-se eficaz na detecção de defeitos precoces de campo visual no glaucoma, relativamente mais sensível e menos específica que a perimetria acromática convencional e a perimetria azul-amarelo. A aplicação de critérios específicos de anormalidade é facilmente transponível à prática clínica. Este exame vem agregar-se ao armamentário semiológico para o diagnóstico funcional precoce do glaucoma, fornecendo informações úteis ao raciocínio clínico, além da facilidade econômica nas situações em que as dificuldades orçamentárias são um empecilho à aquisição de instrumentos derivados de novas tecnologias. 
148 Sensibilidade do estímulo de tamanho I na perimetria computadorizada acromática para a detecção de defeitos glaucomatosos no campo visual: análise comparativa com as perimetrias de ondas curtas e acromática convencional (SITA)

\section{ABSTRACT}

Purpose: To compare sensitivity and specificity of achromatic perimetry with size I stimulus (WW-I) with standard automated perimetry (SITA strategy) and blue on yellow perimetry (SWAP) for early diagnosis of glaucoma. Methods: Seventythree eyes of 73 suspects, glaucomatous and normal subjects underwent automated perimetry with SITA 24-2, WW-I and SWAP. After application of specific criteria of abnormality, sensitivity, specificity, and the area under ROC (Receiver Operating Characteristic) curve were calculated for each of the examinations. The areas under the ROC curve were compared pair-wisely with the univariable $\mathrm{Z}$ test and significance set at 5\%. Results: WW-I displayed the greatest sensitivity (100\%) and the lowest specificity $(75.7 \%)$. SWAP revealed less sensitivity $(69.4 \%)$; specificity, however, was higher (89.2\%). Pairwise comparison of ROC curves showed no difference. Conclusion: WW-I revealed good sensitivity for the detection of early glaucomatous visual field defects. This technique adds to the available armamentarium for early functional diagnosis of glaucomatous optic neuropathy.

Keywords: Perimetry; Glaucoma; Sensitivity and specificity; Visual fields

\section{REFERÊNCIAS}

1. National Advisory Eye Council. Vision Research - A National Plan: 19992003, Executive Summary. Bethesda, MD: National Institute of Health; 1998. [NIH Pub. № 98-4288].
2. Quigley HA, Addicks EM, Green WR. Optic nerve damage in human glaucoma. III. Quantitative correlation of nerve fiber loss and visual field defect in glaucoma, ischemic neuropathy, papilledema, and toxic neuropathy. Arch Ophthalmol. 1982;100(1):135-46.

3. Sommer A, Katz J, Quigley HA, Miller NR, Robin AL, Richter RC, Witt KA. Clinically detectable nerve fiber atrophy precedes the onset of glaucomatous field loss. Arch Ophthalmol. 1991;109(1):77-83.

4. Johnson CA. Recent developments in automated perimetry in glaucoma diagnosis and management. Curr Opin Ophthalmol. 2002;13(2):77-84.

5. Casson R, James B, Rubinstein A, Ali H. Clinical comparison of frequency doubling technology perimetry and Humphrey perimetry. Br J Ophthalmol. 2000;85(3):360-2.

6. Paczka JA, Friedman DS, Quigley HA, Barron Y, Vitale S. Diagnostic capabilities of frequency-doubling technology, scanning laser polarimetry, and the nerve fiber layer photographs to distinguish glaucomatous damage. Am J Ophthalmol. 2001;131(2):188-97.

7. Medeiros FA, Sample PA, Weinreb RN. Frequency doubling technology perimetry abnormalities as predictors of glaucomatous visual field loss. Am J Ophthalmol. 2004;137(5):863-71.

8. Johnson CA, Adams AJ, Casson EJ, Brandt JD. Blue-on-yellow perimetry can predict the development of glaucomatous field loss. Arch Ophthalmol. 1993;111(5):645-50

9. Zalta AH, Burchfield JC. Detecting early glaucomatous field defects with the size I stimulus and Statpac. Br J Ophthalmol. 1990;74(5):289-93.

10. Yamada K, Osako M, Osako S, Okano T, Usui M. The effectiveness of detecting early glaucomatous field defects using the size I stimulus. In: Wall M, Mills RP, editors. Perimetry Update 2000/2001. The Hague: Kugler Publications; 2001. p.51-8.

11. Anderson DR. Automated static perimetry. St. Louis: Mosby Year Book; c1992. p.123

12. Polo V, Larrosa JM, Pinnila I, Pablo L, Honrubia FM. Optimum criteria for short-wavelength automated perimetry. Ophthalmology. 2001;108(2):285-9.

13. Gramer E, Kontic D, Krieglstein GK. [Computer perimetry of glaucomatous visual field defects at different stimulus sizes (author's transl)]. Ophthalmologica. 1981;183(3):162-7. German.

14. Soliman MA, de Jong LA, Ismaeil AA, van den Berg TJ, de Smet MD. Standard achromatic perimetry, short wavelength automated perimetry, and frequency doubling technology for detection of glaucoma damage. Ophthalmology. 2002;109(3):444-54 